\title{
MCR: modern colistin resistance
}

\author{
I. Caniaux ${ }^{1}$ • A. van Belkum ${ }^{2}$ - G. Zambardi ${ }^{2}$ - L. Poirel $^{3,4}$ • M. F. Gros ${ }^{1}$
}

\begin{abstract}
Recently, plasmid-mediated and, therefore, transferable bacterial polymyxin resistance was discovered in strains from both humans and animals. Such a trait may widely spread geographically, while simultaneously crossing microbial species barriers. This may ultimately render the "last resort" polymyxin antibiotics therapeutically useless. Colistin is currently used to treat infections caused by Gram-negative carbapenemase producers and colistin resistance may lead to practical panantibiotic resistance. We here analyzed the medical and diagnostic consequences of (emerging) colistin resistance and propose pathways toward adequate diagnostics for timely detection of both asymptomatic carriage and infection. Culture-based testing using chromogenic and selective media for screening clinical (and veterinary) specimens may constitute key tools for that purpose. Relevant molecular tests are also discussed.
\end{abstract}

\section{What is colistin?}

Colistin, also known as polymyxin E, is produced by Paenibacillus polymyxa and belongs to the polymyxin class of antibiotics. It is a polycationic peptide with both

M. F. Gros

marie-francoise.gros@biomerieux.com

1 Medical Affairs, bioMérieux SA, 376, Chemin de l'Orme, 69280 Marcy L'Etoile, France

2 Scientific Office, bioMérieux SA, 3, Route de Port Michaud, 38390 La Balme-les-Grottes, France

3 Medical and Molecular Microbiology Unit, Department of Medicine, Faculty of Science, University of Fribourg, Rue Albert Gockel 3, 1700 Fribourg, Switzerland

4 INSERM European Unit (LEA, IAME, France), University of Fribourg, Fribourg, Switzerland hydrophilic and lipophilic domains. The cationic regions interact with bacterial lipopolysaccharide (LPS) in the outer cell membrane, where it displaces magnesium and calcium counter ions. Colistin is not homogeneous at the molecular level and consists of a mix of the cyclic polypeptides A and B. Colistin essentially solubilizes the bacterial cell membrane, which is bactericidal in an aqueous environment. It is an older drug with significant nephrotoxicity.

Colistin is commonly used in agriculture in many countries for the control of infections in pigs and, in particular, cattle. Its usage in humans was, so far, rather limited, mainly because of its renal toxicity, but it has been recently re-introduced as a last-line option to treat extensively antibiotic-resistant bacteria such as carbapenem-resistant strains [i.e., NDM-1- and KPCpositive bacterial isolates or extensively drug-resistant (XDR) Acinetobacter baumannii and Pseudomonas aeruginosa]. Colistin has been effective in treating infections caused by P. aeruginosa, Escherichia coli, and Klebsiella spp., for which the range of "normal" minimum inhibitory concentrations (MICs) is $\leq 0.06-4 \mu \mathrm{g} / \mathrm{ml}, 0.12-2 \mu \mathrm{g} / \mathrm{ml}$, and $0.25-2 \mu \mathrm{g} / \mathrm{ml}$ for their wild-type populations, respectively. Colistin is also used for (a) prophylactic and selective decontamination of the digestive tract in intensive care unit (ICU) patients and (b) reducing respiratory tract infections [1]. "Classical" resistance to colistin is rare. However, the SENTRY surveillance network detected colistin resistance associated with the presence of the plasmid-mediated colistin resistance mechanism, involving the $m c r-1$ gene, among $E$. coli and $K$. pneumoniae during 2014-2015 [2-4].

\section{Classical colistin resistance}

Antibiotic susceptibility testing (AST) is normally performed using highly standardized microbiological technology and, in 
the case of colistin, the most common protocol has been jointly endorsed by both the Clinical \& Laboratory Standards Institute (CLSI) and the European Committee on Antimicrobial Susceptibility Testing (EUCAST), the American and European groups overseeing standardization of AST laboratory protocols. Measurement of exact colistin MICs is associated with methodological issues, but the ISOstandard broth microdilution (BMD) method (20776-1) works well for Enterobacteriaceae, P. aeruginosa, and Acinetobacter spp. Specifically, additive-free cation-supplemented MuellerHinton broth needs to be employed. Trays must be made of plain polystyrene and not treated in any way before use, whereas sulfate salts of the antibiotic work best. Susceptibility testing by other methods, including agar dilution, disk diffusion, and gradient diffusion, cannot be recommended in the absence of regional historical colistin MIC data as a reference. Newer methods including the molecular ones are still in need of optimization.

Classical resistance to colistin can be explained by a variety of mechanisms. In E. coli, Salmonella enterica [3], $K$. pneumoniae, and $P$. aeruginosa, the net LPS charge can be reduced upon modification of lipid A through the chemical coupling of phosphoethanolamine or L-amino-arabinose through activation of the $p m r C$ or $p m r F$ operons. Also, the presence of a capsule has been shown to interfere with polymyxin susceptibility in K. pneumoniae. Resistance to colistin in A. baumannii may be associated with mutations in the PmrAB twocomponent system or with a progressive to full loss of LPS through mutations in the $l p x A, l p x C$, and $l p x D$ genes [5]. A single mutation in the PmrB protein, which is a sensor kinase, can lead to the addition of phosphoethanolamine to lipid A, which causes high-level colistin resistance in A. baumannii. Studies in $P$. aeruginosa also suggested a potential role in the resistance to polymyxins for $\mathrm{OprH}$, an outer membrane protein contributing to membrane stability [6]. Multidrug efflux systems can also be responsible for polymyxin resistance in $P$. aeruginosa [7]. Changes in the regulatory loci $p m r A$ and phoP have also been shown to be responsible for polymyxin resistance in Enterobacteriaceae. With time, additional novel resistance mechanisms will be identified. Until very recently, colistin resistance was always thought to be chromosomally encoded allowing vertical transmission only. In addition, this type of resistance often appears to be quite unstable, self-limiting, and does not prevent the successful use of polymyxins.

\section{Emerging mobile colistin resistance markers}

In late 2015, a plasmid-borne $m c r-1$ gene encoding a phosphoethanolamine transferase giving rise to polymyxin resistance was first identified in bacterial strains of animal origin, but also in those cultured from humans and the environment [8]. The plasmid localization of that resistance trait allows for horizontal transmission of the colistin resistance. Surveillance of $E$. coli strains from food animals in China showed that the plasmid was present in $15 \%$ of strains found in meat samples and in $21 \%$ of strains derived directly from animals. It was documented that the strains emerged locally between 2012 and 2014. MCR-1-producing E. coli and $K$. pneumoniae strains were detected in blood and urine specimens from hospitalized patients, showing that host species barriers had already been crossed by the plasmid. In addition, all strains were extended-spectrum $\beta$-lactamase (ESBL) producers [8] and similar strains were isolated from outpatients as well [9].

Transferable colistin resistance has been detected in $10 \%$ of NDM-1-producing carbapenem-resistant Enterobacteriaceae (CRE) in the United Kingdom [10]. In Italy, the proportion of colistin resistance among KPC-producing $K$. pneumoniae was $57 \%$ in 2013 [11]. Following these important findings, several international teams started looking for this new gene in existing collections of bacteria. In January 2016, mcr-1-positive strains were already found in about 19 different countries, including Malaysia [7], France [12, 13], Portugal [14], Laos [15], USA, Thailand, Algeria [16, 17], Germany [18], Switzerland [19, 20], Japan, Belgium, Vietnam [21, 22], the Netherlands [23], Spain [24], and Denmark [25]. The frequency of occurrence of $\mathrm{mcr}-1$ positive bacterial isolates still seems relatively low in humans: in China, $1.4 \%$ of the E. coli strains and $0.7 \%$ of the K. pneumoniae isolates scored phenotypically positive. In Europe (Denmark), $0.2 \%$ of the ESBL- and AmpC-positive E. coli strains are $m c r-1$ PCR-positive. In animals, the detection of $m c r-1$ was lower in Europe than in China, with $15 \%$ of detection in the latter country compared to $1.5 \%$ in the Netherlands and $2 \%$ in Denmark [26].

In bacterial strains from humans, transferable colistin resistance was mainly identified in isolates from patients who were not treated with colistin, and it has been speculated that acquisition of such resistant strains might have resulted from the ingestion of contaminated meat, leading to asymptomatic gastrointestinal colonization [25]. Currently, mcr-1 has been found in E. coli, K. pneumoniae, and Salmonella, and could be successfully maintained in $P$. aeruginosa [8]. Although many mcr-1-positive isolates were shown to co-produce an ESBL enzyme, it is noteworthy that many mcr-1-producing $E$. coli isolates are fully susceptible to $\beta$-lactams and are, therefore, far from multidrug-resistant [13, 23, 25]. Nevertheless, there have been reports of $m c r-1$-producing multidrug-resistant isolates, and, in particular, carbapenemase-producing ones. The first identified isolate was a VIM-1-producing E. coli recovered in Switzerland from a patient who did not have any history of foreign travel [19]. That isolate remained susceptible only to amikacin, fosfomycin, and tigecycline. Recently, an NDM-5- and $m c r$-1-producing $E$. coli has been recovered in the USA, but, still, several drugs were active against that organism 
including amikacin, gentamicin, aztreonam, and nitrofurantoin [27]. In China, some cases of $K$. pneumoniae isolates co-producing $m c r-1$ and NDM-5 have also been reported [9]. An association of $m c r-1, b l a_{\mathrm{NDM}-9}$, and $f o s A 3$ genes has also be reported in $E$. coli strains from chicken products, exhibiting high level of resistance to colistin [28]. The $m c r-1$ gene has been found in KPC-producing and carbapenem-resistant $K$. pneumonia isolates in Italy and Greece as well.

Overall, the occurrence of such multidrug-resistant enterobacterial isolates underscores the importance of this major emerging problem and highlights that care needs to be taken to prevent further dissemination. However, recent studies showed that the occurrence of $\mathrm{mcr}$-producing isolates remains relatively low in many countries. For instance, a very low prevalence of $m c r-1$ and $m c r-1$ producers was found in Switzerland among enterobacterial isolates recovered from urinary tract infections [29]. Similarly, no $\mathrm{mcr}$-1-producing enterobacterial isolate was identified when screening a large collection of enterobacterial isolates recovered from bacteremia in the same country [30]. A significant increase in the detection of colistin resistance has been observed in isolates [22]: up to $50 \%$ of $E$. coli isolated from swine with diarrhea or edema were $m c r-1$-positive. A high frequency of $m c r-1$-positive $E$. coli has been seen in livestock and food isolates, mainly in turkey $(10.7 \%)$ and broilers $(5.6 \%)$ in Germany [4]. Variants of the $m c r-1$ gene have been identified, with $m c r-2$ reported by Xavier et al. [31] in Belgium from porcine and bovine samples, and $m c r-1.2$ by Di Pilato in Italy in K. pneumoniae [32].

\section{Clinical consequences and medical needs}

Continued spread of the plasmid-borne $m c r-1$ gene will compromise the clinical utility of last-resort polymyxins, which may lead to more frequent treatment failure and substantial additional morbidity and mortality. If establishing itself in successful pandemic clones such as E. coli ST131 or K. pneumoniae ST11/ST258, acquisition of $m c r-1$ will likely lead to the global presence of pan-drug-resistant pathogens. Consequently, the European Medicines Agency (EMA), in response to a European Commission request, is currently updating the clinical guidelines on the use of colistin. The EMA is considering to upgrade colistin as an antibiotic of category 2, dedicated to critical medicine and to be used only if no alternatives actually exist. The World Health Organization (WHO) already considers colistin of crucial importance in human medicine (http://who. int/iris/bitstream/10665/77376/1/9789241504485_eng.pdf). The European Centre for Disease Prevention and Control (ECDC) recently released a medical risk assessment on plasmid-mediated colistin resistance in Enterobacteriaceae (ECDC rapid risk assessment. Plasmid-mediated colistin resistance in Enterobacteriaceae, June 13th, 2016; http://www. ecdc.europa.eu). The document describes a short series of specific actions to help reduce colonization and infection risks:

- Develop improved laboratory methods for colistin AST and molecular $m c r-1$ detection. Microbroth dilution is the recommended reference method, while several diagnostic PCR tests have already been described.

- Implement integrated surveillance in hospitals, the community, food, animals, and environment (the One Health approach).

- Screening of multidrug-resistant enterobacterial isolates from patients who recently visited healthcare facilities in foreign countries.

- Perform targeted surveillance to detect reservoirs and include individuals who underwent selective decontamination of the gastrointestinal tract using polymyxins [1].

- Carefully manage patients infected with colistin-resistant strains and reinforce infection prevention and control measures [33].

- Assure that colistin treatment is always accompanied by adequate AST of clinical isolates.

- Apply prudent antimicrobial therapy $[17,34]$ and screening for carriage at hospital admission $[35,36]$.

\section{Recommendations}

Various pragmatic aspects must be considered to help improve the current diagnostic and clinical needs:

The use of selective culture media as an initial screening tool Culture media are well accepted in clinical microbiology and quite well available and affordable on a worldwide scale. The development of novel selective (and possibly chromogenic) culture media will facilitate screening for colonization or infection of high-risk patients: those previously hospitalized, those hospitalized in endemic zones, those with a known history of infection or colonization with colistin-resistant bacteria, or those who were recently exposed to antibiotics. One needs to keep in mind that future work might establish if hospitalization is, indeed, the most significant risk factor since, even to date, several community cases have already been reported. This will eventually help to implement appropriate infection control measures [37, 38]. Such screening should include patients who were previously subjected to colistin-based decolonization [1].

Besides the potential use in human medicine, selective culture media are also relevant to veterinary surveillance considering that screening may soon become a regulatory requirement (e.g., screening of fecal samples from livestock), as part of the ongoing monitoring programs for antibiotic resistance 
in bacteria from animals (http://www.healthcanal. com/infections). To date, the exact prevalence of the $\mathrm{mcr}-1$ gene remains unknown. Benefits of culture-based polymyxin resistance screening and rapid detection of polymyxin resistance in Enterobacteriaceae have been pointed out [39, 40]. To render the search for colistin resistance more clinically useful, simultaneous detection of carbapenem resistance should be considered. A suitable culture medium facilitating such a combined diagnosis could be of significant relevance in countries facing coinciding and possibly extensive spread of carbapenem resistance.

The recently developed Super Polymyxin selective medium provides a useful tool for the screening of polymyxinresistant Gram-negative isolates [39]. Using a large collection of polymyxin-resistant isolates, it has been shown to possess close to $100 \%$ sensitivity and specificity. Noteworthy, it may detect both intrinsic and acquired polymyxin resistance, either using replicates of isolated colonies or even direct stool or rectal swabs samples, depending on local surveillance needs.

Disk diffusion assays, antibiotic gradient testing, and automated AST Disk diffusion methods are not appropriate for the sensitive and specific detection of colistin resistance and, hence, there needs to be a specific search for alternative, fast, reproducible, and, to date, preferably culture-based AST methods. The same problem holds true for gradient testing (e.g., the Etest, which is available in research-use-only format for colistin), which seems to underestimate resistance levels, following evaluation studies conducted by the EUCAST. Currently, only BMD methods are recommended as reliable tests for colistin resistance. However, this method is quite cumbersome and labor-intensive to perform. A reliable colistin MIC test is of utmost importance for patient management, since colistin is a drug with significant adverse effects, which requires precise therapeutic drug monitoring (TDM) to avoid toxic effects while maintaining sufficient antibacterial activity. In addition, precise assessment of serum dosage is important as well, and dosing needs to be compatible with the MIC(s) of the infecting strain(s). Innovative propositions for rapid culture-based testing are still urgently needed. In order to increase the throughput, precision, and speed of colistin resistance testing, it would be good if (additional) assays for the commercially available automated AST systems could be developed or improved. The recently developed Rapid Polymyxin NP test is a tool that is available for accurate distinction between susceptible or resistant strains [39]. It may be implemented worldwide and is affordable. Results are obtained in less than $4 \mathrm{~h}$ and both specificity and susceptibility are high. This colorimetric test may be used for testing colonies and positive blood cultures, therefore providing an interesting tool for the rapid implementation of adequate therapies in the context of bacteremia and sepsis [40]. The interest in rapid confirmation of polymyxin resistance using phenotypic tests has been highlighted by Jayol et al. [41], especially if it can be done directly from blood culture broth in a few hours and enable early adaptation of antimicrobial therapy in case of multidrug resistance.

Molecular diagnostics Matrix-assisted laser desorption/ ionization time-of-flight mass spectrometry (MALDI-TOF MS) can be used for specific protein molecule detection. Currently, however, there is no such test that would directly detect and/or identify the MCR-1 protein, but such tests might be developed, especially if more advanced MS methods could be used. Assays to detect the $m c r-1$ enzymatic activities underlying the resistance or direct detection of the modified LPS molecules might also become interesting options with the progression of MS technology. This may, in the end, still remain difficult to implement diagnostically, since resistance may depend upon rather subtle up- or down-regulation of LPSmodifying enzymes.

Molecular biological detection of the acquired $m c r$-like genes at the nucleic acid level is relatively easy and can be done with simple and straightforward PCR tests, either developed in-house or being part of FDA-approved approaches. Rapid real-time PCR techniques have recently been developed for that purpose and validated using not only pure bacterial cultures, but also stool samples [42, 43]. Such tests could be easily added to existing multiplex panels, usually in addition to genes encoding other key resistance mechanisms. Nevertheless, it is noteworthy that several polymyxin resistance mechanisms are chromosomally encoded and depend upon the substitution of amino acids in diverse regulatory proteins involved in LPS biosynthesis. Therefore, a single PCR approach alone cannot be an adequate tool for an overall diagnosis due to the high diversity of sometimes even coexisting resistance mechanisms. Not only is subsequent sequencing required, but the issue is that correlating amino acid substitutions to corresponding susceptibility or resistance changes (and, hence, distinct MIC values) remains almost impossible.

\section{Conclusion}

Colistin resistance poses a universal problem and rapid detection of the pheno- or genotype is of core importance. Phenotyping would initially be preferred given the diverse, potentially co-existing and complex resistance mechanisms, and since phenotyping takes into account features that are hard to detect by alternative, usually proteomic, genetic, or genomic methodologies [44]. This short review focused on phenotyping, since the many alternative test formats that do exist at the proof of principle level do not (yet) meet quality and validation requirements as imposed by the FDA and other regulatory agencies. Hence, the development of innovative, 
accurate, and rapid tests, although clinically very important, remains quite challenging in the context of polymyxin resistance, owing to the diversity and complexity of the mechanisms involved. Molecular tests cannot be reliably implemented as long as a careful assessment of the correlation between phenotype and genotype has been accurately and precisely performed. This underscores the necessity to reinforce the current fundamental investigations aiming to exactly decipher the fundamental polymyxin resistance mechanisms and their genetic and proteomic bases.

In fact, the renewed interest in polymyxins observed to date reinforces the need to better evaluate the local and international frequency of occurrence of polymyxin-resistant strains, regardless of the underlying resistance mechanisms, whether plasmid or chromosomally encoded. The recent identification in France of a major outbreak caused by OXA-48-producing and colistin-resistant $K$. pneumoniae strongly underscores the relevance of such monitoring [45] and urges toward a real One Health approach investigating not only human infections and colonization, but including research of food, the environment, and animal reservoirs. Finally, the recent observation that colistin resistance may emerge as a consequence of chlorhexidine use [46] again demonstrates the complexity of antibiotic resistance and the need for concerted actions towards the optimal use of antibiotics and antiseptics.

\section{References}

1. Rawson TM, Moore LS, Hatcher JC, Donaldson H, Holmes AH (2016) Plasmid-mediated colistin resistance mechanisms: is it time to revise our approach to selective digestive decontamination? Lancet Infect Dis 16:149-150

2. Castanheira M, Griffin MA, Deshpande LM, Mendes RE, Jones RN, Flamm RK (2016) Detection of $m c r-1$ among Escherichia coli clinical isolates collected worldwide as part of the SENTRY Antimicrobial Surveillance Program in 2014 and 2015. Antimicrob Agents Chemother 60:5623-5624

3. Agersø Y, Torpdahl M, Zachariasen C, Seyfarth A, Hammerum AM, Nielsen EM (2012) Tentative colistin epidemiological cutoff value for Salmonella spp. Foodborne Pathog Dis 9:367-369

4. Irrgang A, Roschanski N, Tenhagen BA, Grobbel M, Skladnikiewicz-Ziemer T, Thomas K, Roesler U, Käsbohrer A (2016) Prevalence of $m c r-1$ in E. coli from livestock and food in Germany, 2010-2015. PLoS One 11, e0159863

5. Beceiro A, Moreno A, Fernández N, Vallejo JA, Aranda J, Adler B, Harper M, Boyce JD, Bou G (2014) Biological cost of different mechanisms of colistin resistance and their impact on virulence in Acinetobacter baumannii. Antimicrob Agents Chemother 58:518526

6. Olaitan AO, Morand S, Rolain JM (2014) Mechanisms of polymyxin resistance: acquired and intrinsic resistance in bacteria. Front Microbiol 5:643

7. Jansen G, Mahrt N, Tueffers L, Barbosa C, Harjes M, Adolph G, Friedrichs A, Krenz-Weinreich A, Rosenstiel P, Schulenburg H (2016) Association between clinical antibiotic resistance and susceptibility of Pseudomonas in the cystic fibrosis lung. Evol Med Public Health 2016:182-194

8. Liu YY, Wang Y, Walsh TR, Yi LX, Zhang R, Spencer J, Doi Y, Tian G, Dong B, Huang X, Yu LF, Gu D, Ren H, Chen X, Lv L, He D, Zhou H, Liang Z, Liu JH, Shen J (2016) Emergence of plasmidmediated colistin resistance mechanism MCR-1 in animals and human beings in China: a microbiological and molecular biological study. Lancet Infect Dis 16:161-168

9. Du H, Chen L, Tang YW, Kreiswirth BN (2016) Emergence of the mcr-1 colistin resistance gene in carbapenem-resistant Enterobacteriaceae. Lancet Infect Dis 16:287-288

10. Jain A, Hopkins KL, Turton J, Doumith M, Hill R, Loy R, Meunier D, Pike R, Livermore DM, Woodford N (2014) NDM carbapenemases in the United Kingdom: an analysis of the first 250 cases. J Antimicrob Chemother 69:1777-1784

11. Giani T, Arena F, Vaggelli G, Conte V, Chiarelli A, Henrici De Angelis L, Fornaini R, Grazzini M, Niccolini F, Pecile P, Rossolini GM (2015) Large nosocomial outbreak of colistin-resistant, carbapenemase-producing Klebsiella pneumoniae traced to clonal expansion of an $m g r B$ deletion mutant. J Clin Microbiol 53:3341-3344

12. Webb HE, Granier SA, Marault M, Millemann Y, den Bakker HC, Nightingale KK, Bugarel M, Ison SA, Scott HM, Loneragan GH (2016) Dissemination of the $m c r-1$ colistin resistance gene. Lancet Infect Dis 16:144-145

13. Haenni M, Poirel L, Kieffer N, Châtre P, Saras E, Métayer V, Dumoulin R, Nordmann P, Madec JY (2016) Co-occurrence of extended spectrum $\beta$ lactamase and MCR-1 encoding genes on plasmids. Lancet Infect Dis 16:281-282

14. Figueiredo R, Henriques A, Sereno R, Mendonça N, da Silva GJ (2015) Antimicrobial resistance and extended-spectrum $\beta$ lactamases of Salmonella enterica serotypes isolated from livestock and processed food in Portugal: an update. Foodborne Pathog Dis 12:110-117

15. Olaitan AO, Thongmalayvong B, Akkhavong K, Somphavong S, Paboriboune P, Khounsy S, Morand S, Rolain JM (2015) Clonal transmission of a colistin-resistant Escherichia coli from a domesticated pig to a human in Laos. J Antimicrob Chemother 70:34023404

16. Olaitan AO, Chabou S, Okdah L, Morand S, Rolain JM (2016) Dissemination of the $m c r-1$ colistin resistance gene. Lancet Infect Dis 16:147

17. Olaitan AO, Morand S, Rolain JM (2016) Emergence of colistinresistant bacteria in humans without colistin usage: a new worry and cause for vigilance. Int J Antimicrob Agents 47:1-3

18. Falgenhauer L, Waezsada SE, Yao Y, Imirzalioglu C, Käsbohrer A, Roesler U, Michael GB, Schwarz S, Werner G, Kreienbrock L, Chakraborty T, RESET consortium (2016) Colistin resistance gene mcr- 1 in extended-spectrum $\beta$-lactamase-producing and carbapenemase-producing Gram-negative bacteria in Germany. Lancet Infect Dis 16:282-283

19. Poirel L, Kieffer N, Liassine N, Thanh D, Nordmann P (2016) Plasmid-mediated carbapenem and colistin resistance in a clinical isolate of Escherichia coli. Lancet Infect Dis 16:281

20. Poirel L, Hombrouck-Alet C, Freneaux C, Bernabeu S, Nordmann P (2010) Global spread of New Delhi metallo- $\beta$-lactamase 1. Lancet Infect Dis 10:832

21. Malhotra-Kumar S, Xavier BB, Das AJ, Lammens C, Hoang HT, Pham NT, Goossens H (2016) Colistin-resistant Escherichia coli harbouring $m c r-1$ isolated from food animals in Hanoi, Vietnam. Lancet Infect Dis 16:286-287

22. Kusumoto M, Ogura Y, Gotoh Y, Iwata T, Hayashi T, Akiba M (2016) Colistin-resistant $m c r-1$-positive pathogenic Escherichia coli in swine, Japan, 2007-2014. Emerg Infect Dis 22:1315-1317

23. Arcilla MS, van Hattem JM, Matamoros S, Melles DC, Penders J, de Jong MD, Schultsz C; COMBAT consortium (2016) 
Dissemination of the $m c r-1$ colistin resistance gene. Lancet Infect Dis 16:147-149

24. Prim N, Rivera A, Rodríguez-Navarro J, Español M, Turbau M, Coll P, Mirelis B (2016) Detection of $m c r-1$ colistin resistance gene in polyclonal Escherichia coli isolates in Barcelona, Spain, 2012 to 2015. Euro Surveill 21(13)

25. Hasman H, Hammerum AM, Hansen F, Hendriksen RS, Olesen B, Agersø Y, Zankari E, Leekitcharoenphon P, Stegger M, Kaas RS, Cavaco LM, Hansen DS, Aarestrup FM, Skov RL (2015) Detection of $m c r-1$ encoding plasmid-mediated colistin-resistant Escherichia coli isolates from human bloodstream infection and imported chicken meat, Denmark 2015. Euro Surveill 20(49)

26. Kluytmans-van den Bergh MF, Huizinga P, Bonten MJ, Bos M, De Bruyne K, Friedrich AW, Rossen JW, Savelkoul PH, Kluytmans JA (2016) Presence of $m c r-1$-positive Enterobacteriaceae in retail chicken meat but not in humans in the Netherlands since 2009. Euro Surveill 21(9)

27. Mediavilla JR, Patrawalla A, Chen L, Chavda KD, Mathema B, Vinnard C, Dever LL, Kreiswirth BN (2016) Colistin- and carbapenem-resistant Escherichia coli harboring $m c r-1$ and $b l a_{\mathrm{NDM}-5}$, causing a complicated urinary tract infection in a patient from the United States. MBio 7, e01191-16

28. Yao X, Doi Y, Zeng L, Lv L, Liu JH (2016) Carbapenem-resistant and colistin-resistant Escherichia coli co-producing NDM-9 and MCR-1. Lancet Infect Dis 16:288-289

29. Liassine N, Assouvie L, Descombes MC, Tendon VD, Kieffer N, Poirel L, Nordmann P (2016) Very low prevalence of MCR-1/ MCR-2 plasmid-mediated colistin resistance in urinary tract Enterobacteriaceae in Switzerland. Int J Infect Dis 51:4-5

30. Nordmann P, Assouvie L, Prod'Hom G, Poirel L, Greub G (2016) Screening of plasmid-mediated MCR-1 colistin-resistance from bacteremia. Eur J Clin Microbiol Infect Dis 35(11):1891-1892

31. Xavier BB, Lammens C, Ruhal R, Kumar-Singh S, Butaye P, Goossens H, Malhotra-Kumar S (2016) Identification of a novel plasmid-mediated colistin-resistance gene, mcr-2, in Escherichia coli, Belgium, June 2016. Euro Surveill 21(27)

32. Di Pilato V, Arena F, Tascini C, Cannatelli A, Henrici De Angelis L, Fortunato S, Giani T, Menichetti F, Rossolini GM (2016) $m c r$-1.2, a new $m c r$ variant carried on a transferable plasmid from a colistinresistant KPC carbapenemase-producing Klebsiella pneumoniae strain of sequence type 512. Antimicrob Agents Chemother 60: $5612-5615$

33. Nordmann P, Poirel L (2016) Plasmid-mediated colistin resistance: an additional antibiotic resistance menace. Clin Microbiol Infect 22:398400
34. van Duin D, Doi Y (2015) Outbreak of colistin-resistant, carbapenemase-producing Klebsiella pneumoniae: are we at the end of the road? J Clin Microbiol 53:3116-3117

35. Gray JW, Mahida N (2016) How do you solve a problem like multidrug-resistant Gram-negative bacteria? J Hosp Infect 92:1-2

36. Skov RL, Monnet DL (2016) Plasmid-mediated colistin resistance ( $m c r-1$ gene): three months later, the story unfolds. Euro Surveill 21(9)

37. McGann P, Snesrud E, Maybank R, Corey B, Ong AC, Clifford R, Hinkle M, Whitman T, Lesho E, Schaecher KE (2016) Escherichia coli harboring $m c r-1$ and blaCTX-M on a novel IncF plasmid: first report of $m c r-1$ in the United States. Antimicrob Agents Chemother 60:4420-4421

38. Yu H, Qu F, Shan B, Huang B, Jia W, Chen C, Li A, Miao M, Zhang X, Bao C, Xu Y, Chavda KD, Tang YW, Kreiswirth BN, Du H, Chen L (2016) Detection of the $m c r-1$ colistin resistance gene in carbapenem-resistant Enterobacteriaceae from different hospitals in China. Antimicrob Agents Chemother 60:5033-5035

39. Nordmann P, Jayol A, Poirel L (2016) Rapid detection of polymyxin resistance in Enterobacteriaceae. Emerg Infect Dis 22:1038-1043

40. Nordmann P, Jayol A, Poirel L (2016) A universal culture medium for screening polymyxin-resistant Gram-negative isolates. J Clin Microbiol 54:1395-1399

41. Jayol A, Dubois V, Poirel L, Nordmann P (2016) Rapid detection of polymyxin-resistant Enterobacteriaceae from blood cultures. J Clin Microbiol 54:2273-2277

42. Bontron S, Poirel L, Nordmann P (2016) Real-time PCR for detection of plasmid-mediated polymyxin resistance $(\mathrm{mcr}-1)$ from cultured bacteria and stools. J Antimicrob Chemother 71:2318-2320

43. Nijhuis RH, Veldman KT, Schelfaut J, Van Essen-Zandbergen A, Wessels E, Claas EC, Gooskens J (2016) Detection of the plasmidmediated colistin-resistance gene $m c r-1$ in clinical isolates and stool specimens obtained from hospitalized patients using a newly developed real-time PCR assay. J Antimicrob Chemother 71:2344-2346

44. Schwarz S, Johnson AP (2016) Transferable resistance to colistin: a new but old threat. J Antimicrob Chemother 71:2066-2070

45. Jayol A, Poirel L, Dortet L, Nordmann P (2016) National survey of colistin resistance among carbapenemase-producing Enterobacteriaceae and outbreak caused by colistin-resistant OXA-48-producing Klebsiella pneumoniae, France, 2014. Euro Surveill 21:(37)

46. Wand ME, Bock LJ, Bonney LC, Sutton JM (2016) Mechanisms of increased resistance to chlorhexidine and cross-resistance to colistin following exposure of Klebsiella pneumoniae clinical isolates to chlorhexidine. Antimicrob Agents Chemother (in press) 